\title{
Identification of biomarkers and potential molecular mechanisms of clear cell renal cell carcinoma
}

\author{
F. $\mathrm{WU}^{1}, \mathrm{~S} . \mathrm{WU}^{2}, \mathrm{X} . \mathrm{GOU}^{1, *}$ \\ ${ }^{1}$ Department of Urology, ${ }^{2}$ Department of Gastroenterology, The First Affiliated Hospital of Chongqing Medical University, Chongqing 400016, \\ China. \\ *Correspondence: gouxincq@163.com
}

Received May 11, 2017/ Accepted June 19, 2017

\begin{abstract}
Clear cell renal cell carcinoma (ccRCC) is the most common type of renal cancer in adults. The aim of this study is to identify the biomarkers and potential molecular mechanisms of ccRCC. Three gene expression profiles and two miRNA expression profiles were downloaded from GEO database. A total of 330 up-regulated differentially expressed genes (DEGs), 545 down-regulated DEGs, 26 up-regulated differentially expressed miRNAs (DEMs) and 11 down-regulated DEMs were identified by GEO2R. The gene ontology (GO) enrichment and Kyoto Encyclopedia of Genes and Genomes (KEGG) pathway analysis were performed by KOBAS software. The results showed that GO terms of the up-regulated DEGs were mostly enriched in response to stimulus at BP level, cell periphery at CC level and binding at MF level, while the GO terms of down-regulated DEGs were enriched in single-organism process at BP level, extracellular exosome at CC level and catalytic activity at MF level. As for KEGG pathways, HIF-1 signaling pathway, focal adhesion, PI3K-Akt signaling pathway and metabolic pathways were significantly enriched. Then, protein-protein interaction (PPI) network and miRNA-gene network were constructed and analyzed by Cytoscape. A total of eight DEGs were identified as biomarkers, including VEGFA, PPARA, CCND1, FLT1, CXCL12, FN1, DCN and ERBB4. Expression validation and survival analysis were performed by GEPIA and OncoLnc, respectively. Four biomarkers were verified by quantitative real-time PCR (qPCR) in 786-O cell line and HK-2 cell line. All four genes had the same expression trend as predicted. Our study provides a series of biomarkers and molecular mechanisms for the deeper research of ccRCC.
\end{abstract}

Key words: clear cell renal cell carcinoma, differentially expressed gene, protein-protein interaction network, miRNA, survival analysis

Clear cell renal cell carcinoma (ccRCC) is the most common type of RCC that occurs in adults and has the worst prognosis among the common epithelial tumors of the kidney [1]. Recently, increasingly accurate knowledge on the molecular pathogenesis of this tumor has provided different treatments of RCC [2]. However, the early diagnosis and therapy of ccRCC remain challenging.

Molecular biomarkers have been shown to aid the diagnosis and therapy for many cancers, and some biomarkers are also found in ccRCC [3]. For example, IL13RA2 has been reported to play a role in the acquired sunitinib-resistance in ccRCC [4]. Besides, higher SK1 levels in ccRCC can induce invasion and angiogenesis and lead to shorter survival [5]. Except for gene biomarkers, some miRNAs have also been confirmed as biomarkers of ccRCC. miR-106b-5p can induce cell proliferation and suppress processing of caspase- 3 and cell apoptosis in ccRCC cells by down-regulating SETD2 [6]. However, miR-126 is down-regulated in metastatic ccRCC, and is associated with cellular migration and proliferation [7]. For cancers, it is common that multiple molecules play a role in the same cancer. So, a systemic analysis of the molecular mechanism is needed.

Bioinformatics analysis is a useful tool to perform systemic analysis of diseases. By analyzing gene ontology (GO) terms, we can better understand the biological process (BP), cellular component (CC) and molecular function (MF) of ccRCC [8]. Meanwhile, Kyoto Encyclopedia of Genes and Genomes (KEGG) pathway analysis can provide us the most significant pathways in ccRCC [9]. Besides, network analysis is the best way to identify the biomarkers and molecular interactions [10]. 


\section{Materials and methods}

Microarray data. Microarray profiles were downloaded from the Gene Expression Omnibus (GEO) database (http:// www.ncbi.nlm.nih.gov/geo) [11], including three gene expression profiles (GSE16441, GSE53757 and GSE68417) and two miRNA expression profiles (GSE12105 and GSE16441). The GSE16441 datasets contained 17 ccRCC samples and 17 normal renal samples in both gene and miRNA expression profiles. GSE53757 consisted of 72 ccRCC samples and 72 normal samples. GSE68417 included $29 \mathrm{ccRCC}$ samples and 20 normal samples. GSE12105 contained 12 ccRCC samples and 12 normal samples.

Identification of DEGs and DEMs. The identification of DEGs and DEMs was performed by GEO2R (http://www.ncbi. nlm.nih.gov/geo/info/geo2r.html). GEO2R is a useful web tool for comparing groups of samples to identify differentially expressed genes and miRNAs across experimental conditions. The $\mathrm{p}$-value $<0.05$ and $|\log \mathrm{FC}|>1$ were set as cut-off criteria.

Functional enrichment analysis of DEGs. GO and KEGG analysis are common useful methods for annotating gene functions and identifying biological attributes for genome or transcriptome data. The GO and KEGG pathway analysis were performed by KOBAS 3.0 (http://kobas.cbi.pku.edu.cn) [12] web tool. The p-value $<0.05$ was defined as the cut-off criterion.

PPI network construction and modules analysis. Search Tool for the Retrieval of Interacting Genes (STRING, http:// string.embl.de) database is an online tool to evaluate the protein-protein interaction (PPI) information. To analyze the relationships among the DEGs, PPI network was constructed by STRING (version 10.0), and the combined score $>0.4$ was chosen as the cut-off criterion. Then, PPI network was visualized by Cytoscape (version 3.4.0) and the modules was analyzed by the Molecular Complex Detection (MCODE) app. The cut-off criterion was defined as follows: MCODE scores $>3$ and the number of nodes $>4$. In addition, the KEGG pathway analysis of genes in the modules was performed by KOBAS and the p-value $<0.05$ and input number $>3$ were set as the cut-off criterion.

Prediction of miRNA targets. The prediction of the target genes of DEMs was performed by five established miRNA target prediction programs (miRanda, MirTarget2, PicTar, PITA and TargetScan). The target genes predicted by at least three programs were considered as the targets of the DEMs.

Expression validation and survival analysis. Gene expression profiling interactive analysis (GEPIA, http:// gepia.cancer-pku.cn) [13] was used for validating the expression of hub genes. Survival analysis for mRNAs and miRNAs was performed by OncoLnc (http://www.oncolnc.org).

Cell culture. The human RCC cell line 786-O and normal renal cell line HK-2 were obtained from the Molecular Oncology and Epigenetics Laboratory (the First Affiliated Hospital of Chongqing Medical University, Chongqing, China). The 786-O cell line was cultured in RPMI-1640 medium (Hyclone) supplemented with 10\% fetal bovine serum (PAN), while the HK-2 cell line was cultured in Keratinocyte Serum Free Medium (GIBCO) supplemented with $0.05 \mathrm{mg} / \mathrm{ml} \mathrm{BPE}$ and $5 \mathrm{ng} / \mathrm{ml} \mathrm{EGF}$. Both cell lines were cultured at $37^{\circ} \mathrm{C}$ in $5 \% \mathrm{CO}_{2}$.

Quantitative real-time PCR validation. Total RNA of cultured cells (786-O and HK-2) was extracted by RNAiso Plus (TaKaRa, China). Then, RNA $(1 \mu \mathrm{g})$ was reverse transcribed using GoScript ${ }^{\mathrm{TM}}$ Reverse Transcription System (Promega, USA). Quantitative real-time PCR was performed by GoTaq qPCR Master Mix (Promega, USA) and qPCR values of each gene were normalized against ACTB. Then, the relative expression of genes was calculated by the $2^{-\Delta \Delta \mathrm{Ct}}$ method [14].

Statistical analysis. The qPCR data was presented as mean \pm SD and analyzed by Student's t-test. The p-value $<0.05$ was considered to have statistical differences.

\section{Results}

Identification of DEGs and DEMs. A total of 1763, 2121 and 670 up-regulated DEGs were identified with the 2275, 2442 and 1115 down-regulated DEGs from GSE16441, GSE53757 and GSE68417, respectively. Moreover, 102 and 58 up-regulated DEMs were identified with the 193 and 34 down-regulated DEMs from GSE12105 and GSE16441, respectively. A total of 330 up-regulated DEGs (Figure 1A) and 545 down-regulated DEGs (Figure 1B) were found in the overlap of the three gene expression datasets. Besides, there were 26 up-regulated DEMs (Figure 1C) and 11 downregulated DEMs (Figure 1D) in the overlap of the two miRNA expression datasets.

Functional enrichment analysis of DEGs. The functional and pathway enrichment analysis of the DEGs was performed by KOBAS online tool. GO enrichment results showed that up-regulated DEGs were enriched in response to stimulus, immune system process and single-organism cellular process at BP level; cell periphery, plasma membrane and membrane at $\mathrm{CC}$ level and binding, protein binding and receptor binding at MF level. Down-regulated DEGs were significantly enriched in single-organism process, single-organism metabolic process and organic acid metabolic process at BP level; extracellular exosome, extracellular vesicle and extracellular organelle at CC level and catalytic activity, binding and oxidoreductase activity at MF level (Figure 2). Most GO terms were enriched in the signal transduction and cellular regulation processes and supported the reliability of our analysis.

KEGG pathway analysis revealed that up-regulated DEGs were significantly enriched in HIF-1 signaling pathway, focal adhesion and PI3K-Akt signaling pathway (Figure 3A), while down-regulated DEGs were enriched in metabolic pathways, such as carbon metabolism, propanoate metabolism and fatty acid degradation (Figure $3 \mathrm{~B}$ ).

PPI network construction and modules analysis. The PPI network was analyzed by STRING and constructed by Cytoscape (Figure 4). Then, the modules in the PPI network 

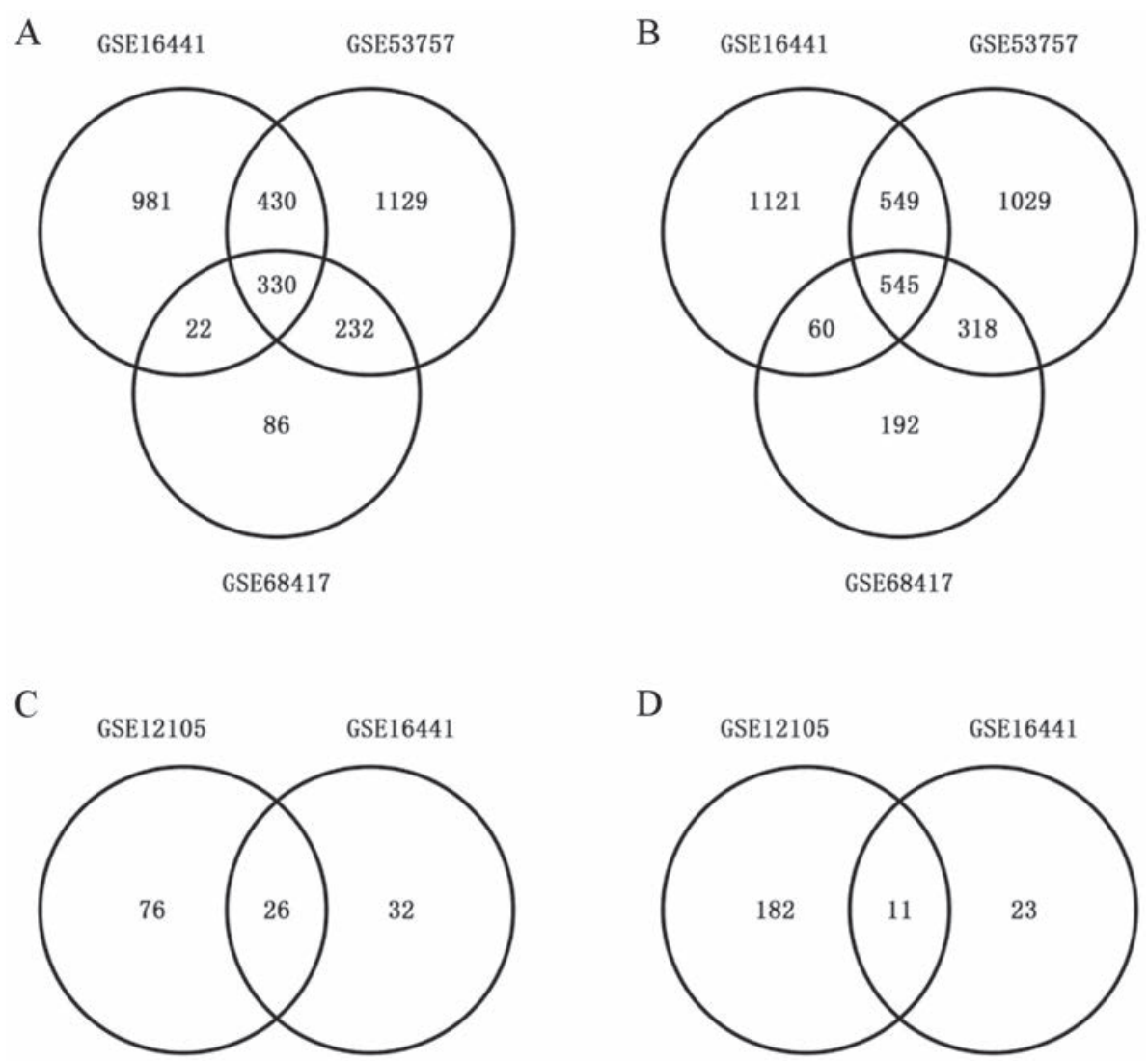

Figure 1. Identification of DEGs and DEMs. (A) The identification of up-regulated DEGs. (B) The identification of down-regulated DEGs. (C) The identification of up-regulated DEMs. (D) The identification of down-regulated DEMs.

were analyzed by MCODE app. The top four modules were chosen and the KEGG pathway analysis of the genes in the top four modules was performed by KOBAS. The pathway enrichment analysis showed that genes in these modules were significantly enriched in HIF-1 signaling pathway, oxidative phosphorylation, metabolic pathways and fatty acid metabolism (Figure 5).

miRNA-gene network. The target genes of the identified DEMs were predicted and compared with DEGs. Then, the overlapping genes were chosen as the target genes. Moreover, the miRNA-gene network was constructed by Cytoscape (Figure 6). To better identify the gene biomarkers in ccRCC, we combined both PPI and miRNA-gene networks. Only the genes meeting the criterion that degree $\geq 30$ in PPI network and degree $\geq 3$ in miRNA-gene network were chosen as the key gene biomarkers. A total of eight gene biomarkers were identified, including VEGFA, PPARA, CCND1, FLT1, CXCL12, FN1, DCN and ERBB4 (Figure 7). Besides, several miRNAs were also identified, such as hsa-miR-142-3p and has-miR-142-5p.

Expression validation and survival analysis. The expression of eight identified hub genes was validated by GEPIA. Four genes (CCND1, FLT1, FN1 and VEGFA) were increased in ccRCC tissues, while three genes (CXCL12, DCN and ERBB4) were decreased. However, the expression of PPARA showed no significant differences between ccRCC tissues and normal tissues (Figure 8). Survival analysis for mRNAs showed that CCND1, DCN, FLT1 and PPARA play important roles in ccRCC. Moreover, hsa-miR-142-3p and has-miR-142-5p also have an influence on the survival of ccRCC patients (Figure 9).

Quantitative real-time PCR validation. To confirm the reliability of the bioinformatics analysis for identifying biomarkers, quantitative real-time PCR was performed. The expression of the four identified gene biomarkers was detected in ccRCC cell line (786-O) and normal renal cell line (HK-2). The above analysis revealed that CCND1 and FN1 were up-regulated DEGs in ccRCC, while ERBB4 and PPARA were down-regulated DEGs. The qPCR results showed that the expression of CCND1 was higher in 786-O cell line than that in HK-2 cell line $(p<0.01)$. The expression of FN1 was higher in 786-O cell line than that in HK-2 cell line $(\mathrm{p}<0.05)$. However, the expression of ERBB4 and PPARA was lower in $786-\mathrm{O}$ cell line than that in HK-2 cell line $(\mathrm{p}<0.05$ and $\mathrm{p}<0.001$, respectively) (Figure 10 ). These results supported the bioinformatics analysis in our study. 

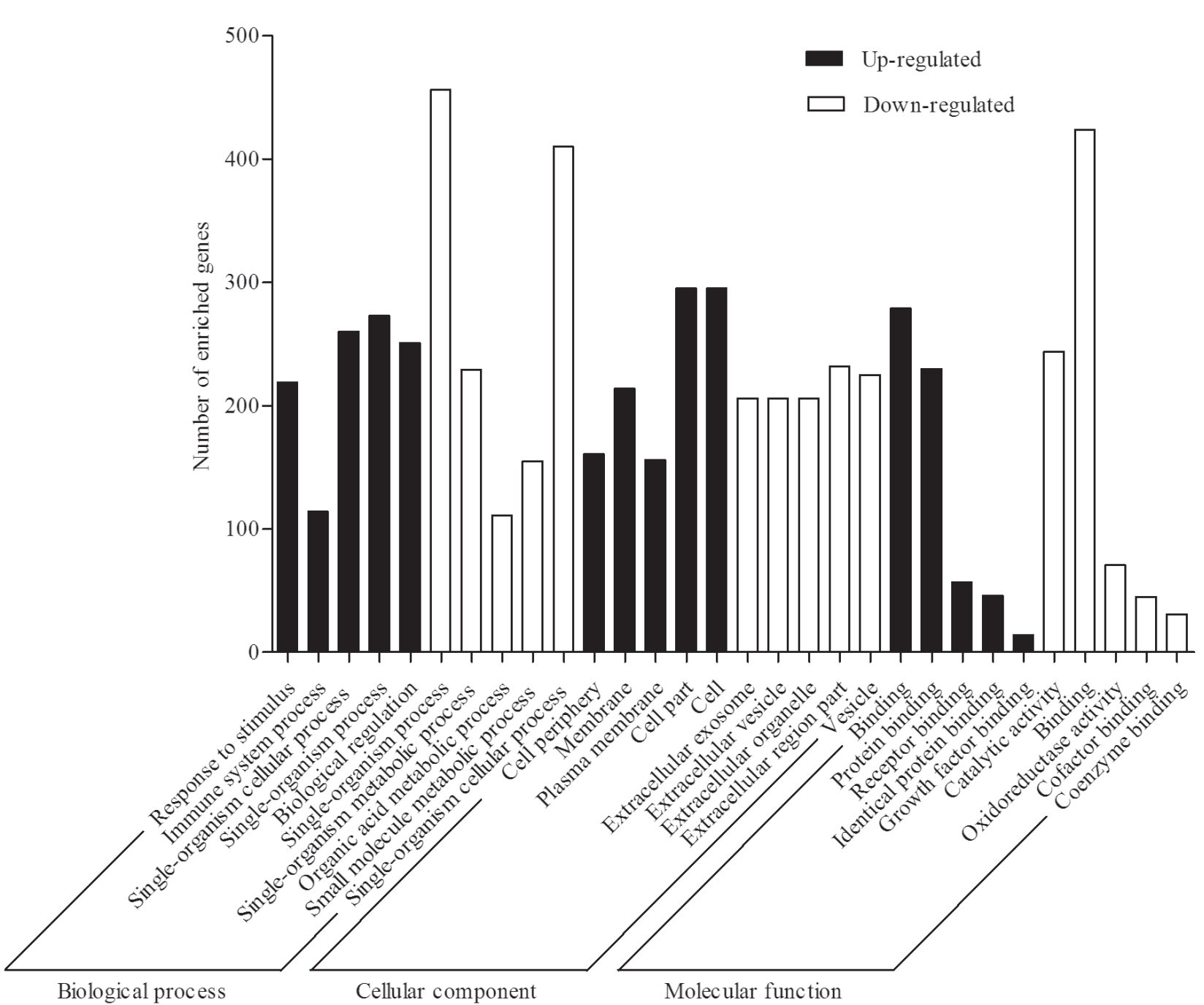

Figure 2. GO terms analysis of the DEGs. Black bars stand for the up-regulated DEGs, while white bars stand for the down-regulated DEGs.
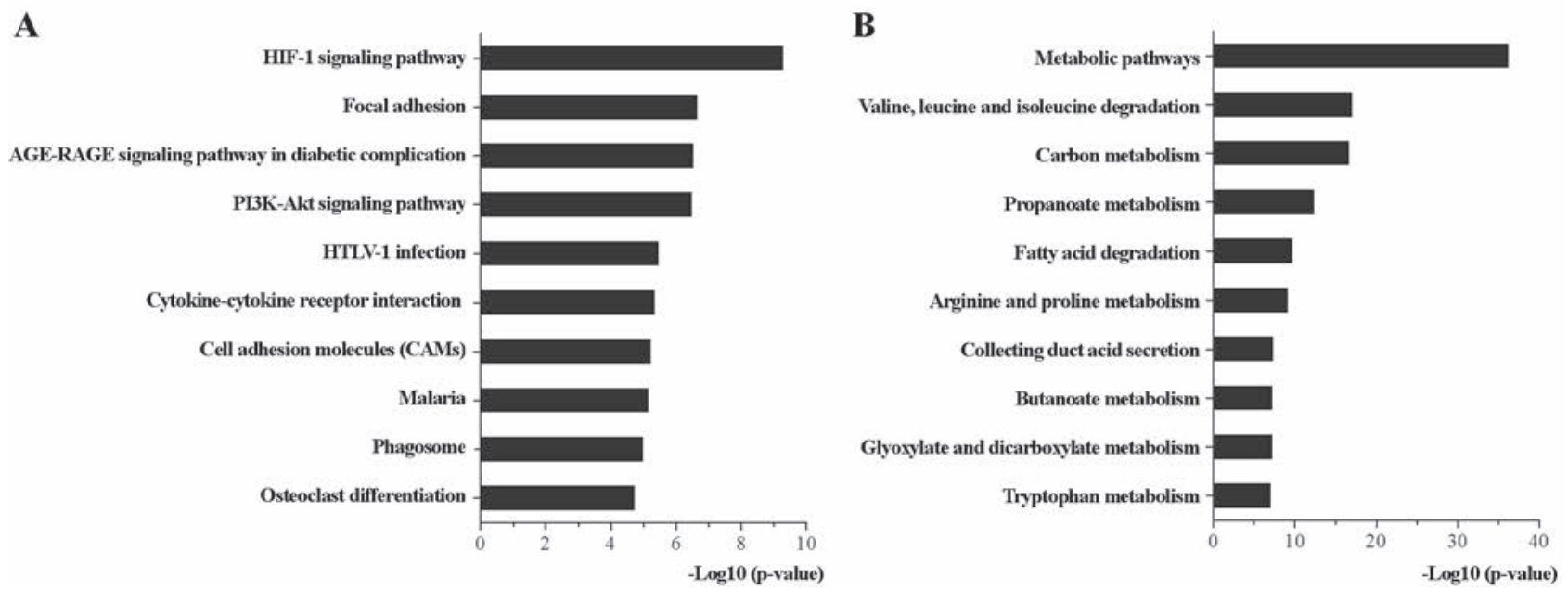

Figure 3. KEGG pathways enrichment of the DEGs. (A) KEGG pathways analysis of the up-regulated DEGs. (B) KEGG pathways analysis of the downregulated DEGs. 


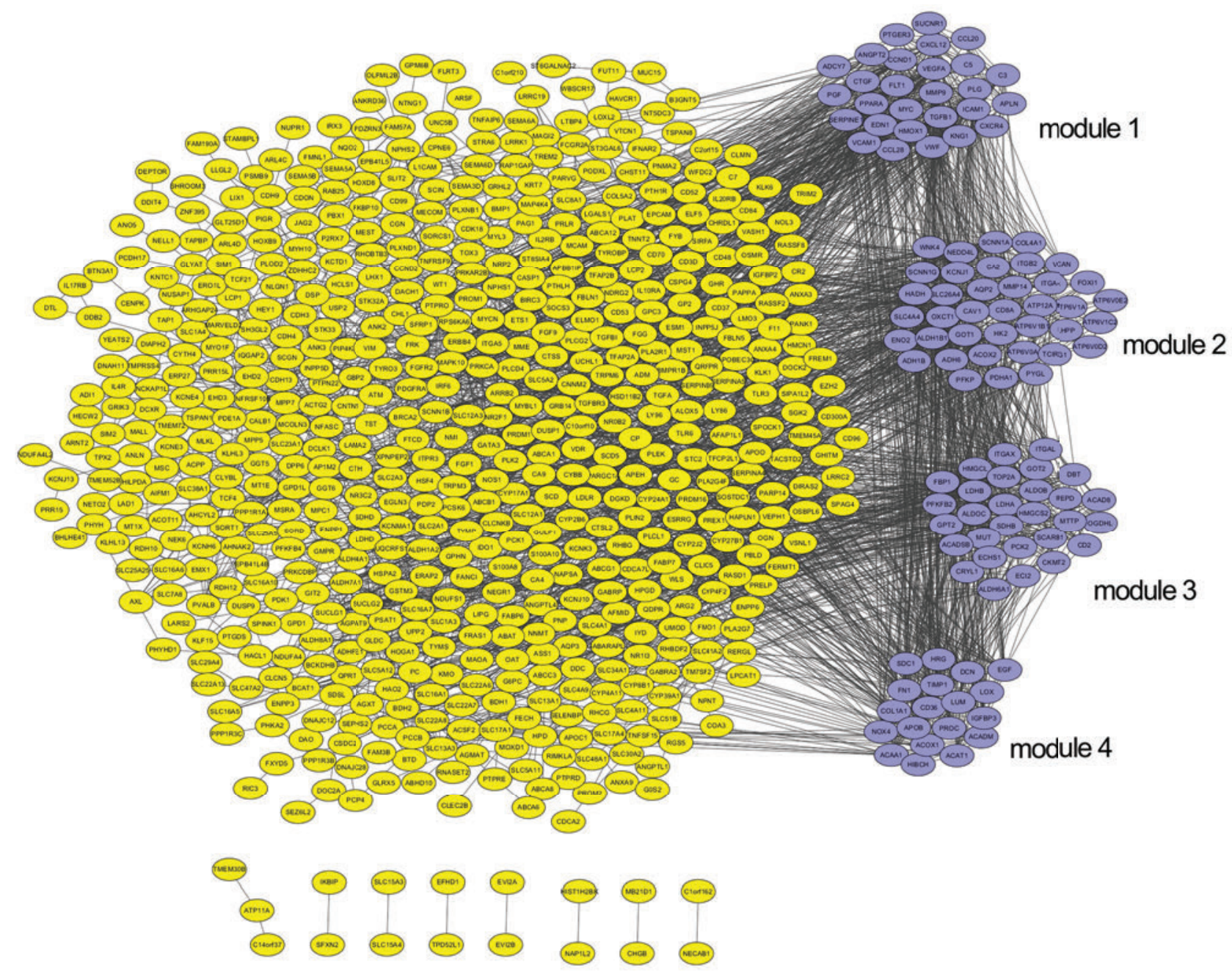

Figure 4. PPI network construction and modules analysis. The yellow nodes represent the DEGs, purple nodes represent the genes involved in the top four modules and the lines represent the interaction between two nodes.

\section{Discussion}

ccRCC is the most common type of RCC in adults. In this study, bioinformatics analysis was performed to identify the biomarkers and potential mechanisms in ccRCC. A total of 330 up-regulated DEGs, 545 down-regulated DEGs, 26 up-regulated DEMs and 11 downregulated DEMs were identified. For the up-regulated DEGs, GO terms were mostly enriched in response to stimulus at BP level, cell periphery at CC level and binding at MF level. For the down-regulated DEGs, GO terms were enriched in single-organism process at BP level, extracellular exosome at CC level and catalytic activity at MF level. Most of these

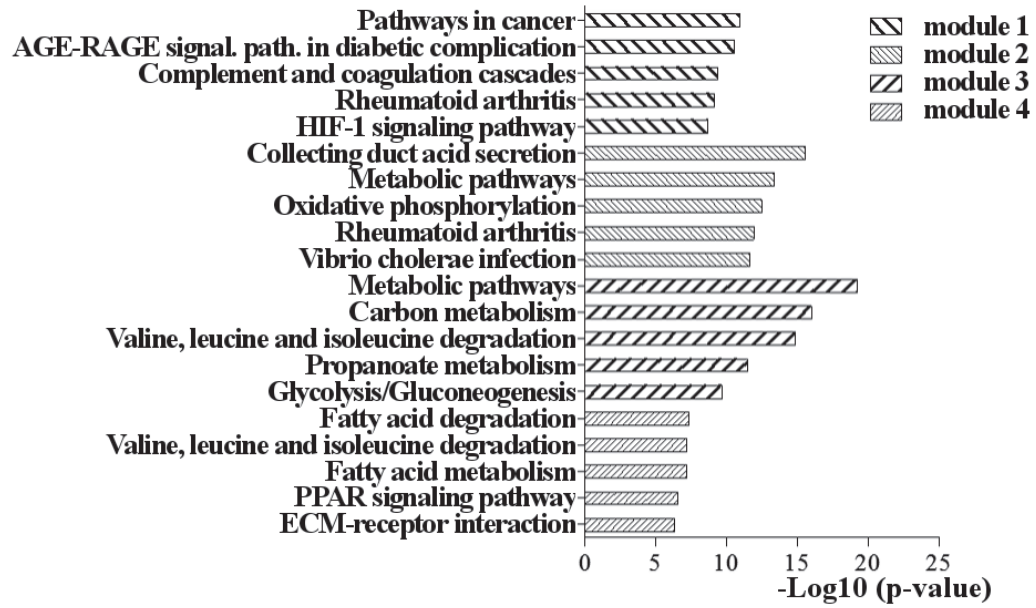

Figure 5. KEGG pathways analysis of the genes in the top four modules. 
enriched GO terms were fundamental concepts of regulatory process in the cell.

KEGG pathway analysis showed that HIF-1 signaling pathway, focal adhesion, PI3K-Akt signaling pathway and metabolic pathways were significantly enriched. Hypoxia grows up in most solid tumors due to incapacity of the existing vascular system to provide the growing tumor mass with adequate amounts of oxygen. Oxidative stress in the tumor stroma imitates the effects of hypoxia resulting in an excess production of ROS. Then, excess ROS activates the antioxidant defense protecting the cancer cells from apoptosis [15]. HIF-1 has been shown to participate in many molecular events required for the adaptation of tumor cells to hypoxia [16]. Besides, ROS and NO can potently trigger activation of HIF-1a leading to hypoxic signaling, such as increased VEGF production and enhanced glycolysis [17]. Metabolic reprogramming is considered as a symbol of cancer [18]. Oncogenic signaling pathways directly enhances nutrient acquisition and facilitates assimilation of carbon leading to support for cell growth and proliferation, such as HIF-1a, PI3K and KRAS signal pathways [19]. The PI3K-Akt pathway is the primary effector of insulin signaling and the activation of Akt promotes glucose uptake, glycolytic flux and lactate excretion [20]. In addition, both Ras activation and hypoxia can activate HIF-1 [21]. These pathways supported the cancer cell growth and proliferation.

The PPI network was constructed and analyzed by Cytoscape software. Pathways analysis of the genes in the

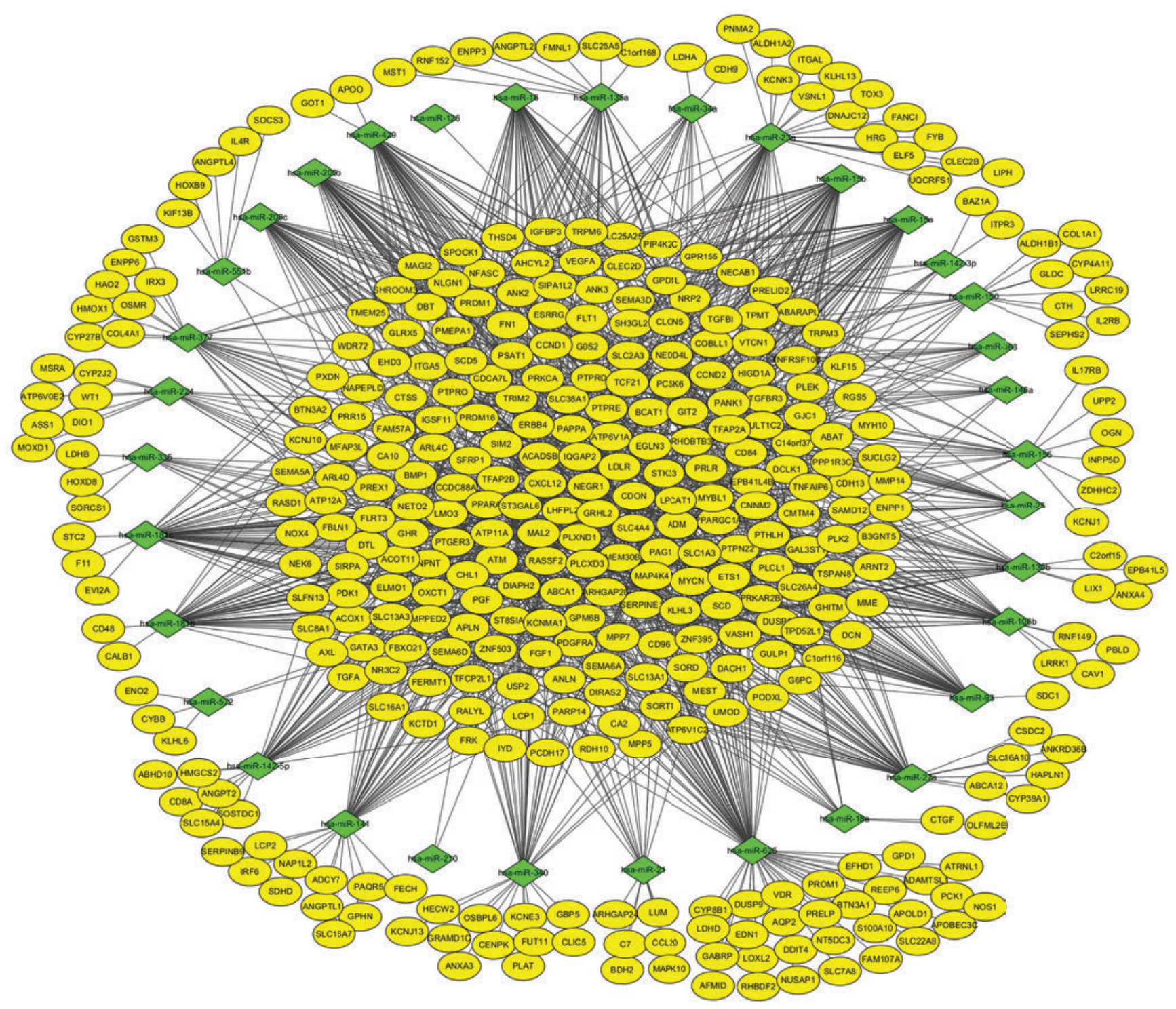

Figure 6. miRNA-gene network. Yellow nodes stand for the DEGs, while green nodes stand for the DEMs. The lines stand for the regulation relationship between DEMs and DEGs. 
top four modules was performed by KOBAS. The results showed that the KEGG pathways were also significantly enriched in HIF-1 signaling pathway, oxidative phosphorylation, metabolic pathways and fatty acid metabolism, which supported the above analysis and could reveal the molecular mechanisms of ccRCC.
miRNAs are series of small non-coding RNA which play a role in regulating cellular processes. By combined analysis of PPI and miRNA-gene network, eight gene biomarkers were identified, including VEGFA, PPARA, CCND1, FLT1, CXCL12, FN1, DCN and ERBB4. VEGFA is a member of the PDGF/VEGF growth factor family. It can induce prolif-

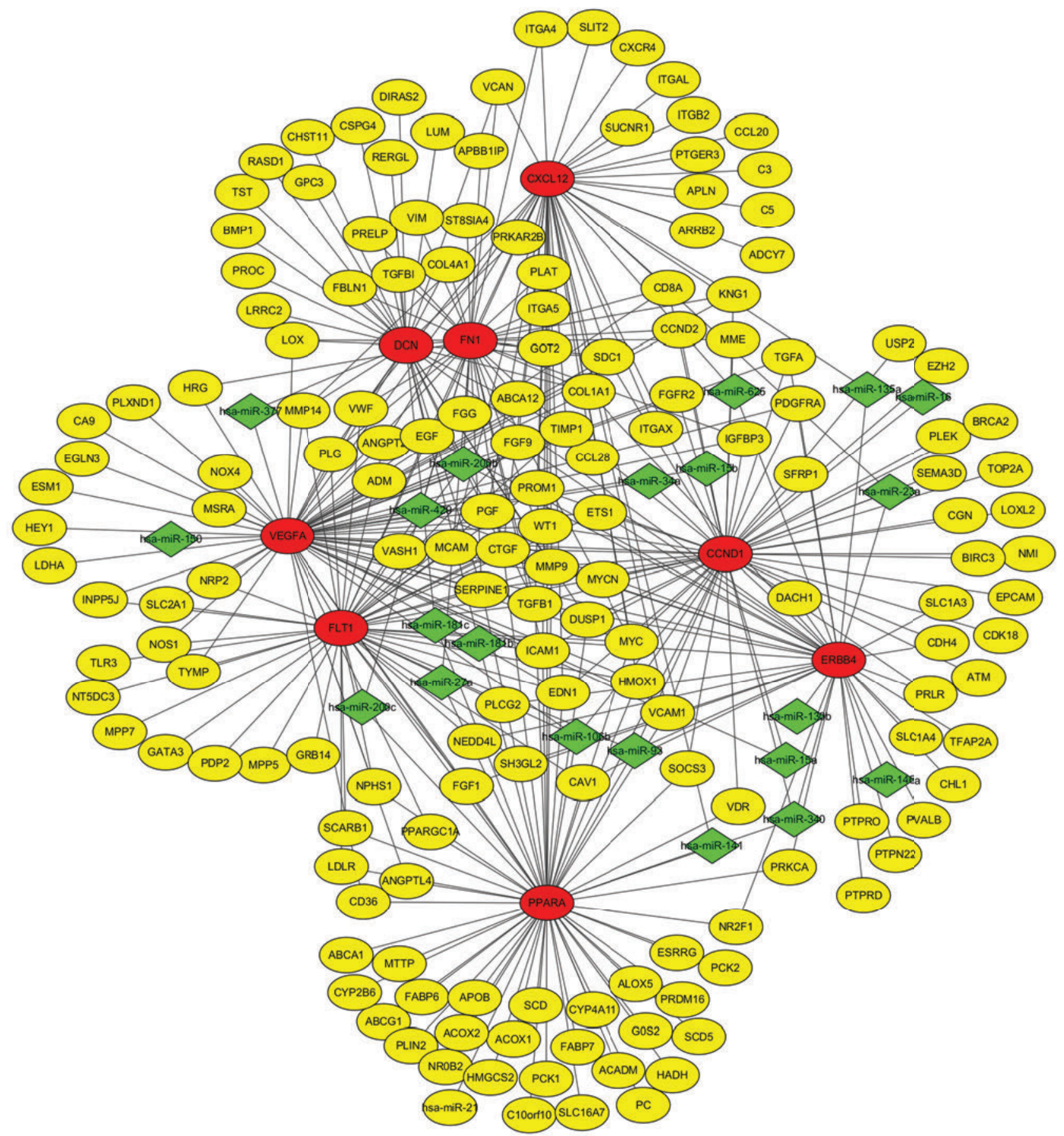

Figure 7. Gene biomarkers regulation network. Yellow nodes stand for the DEGs, red nodes stand for the eight gene biomarkers and green nodes stand for DEMs. The lines stand for the regulation relationship between two nodes. 

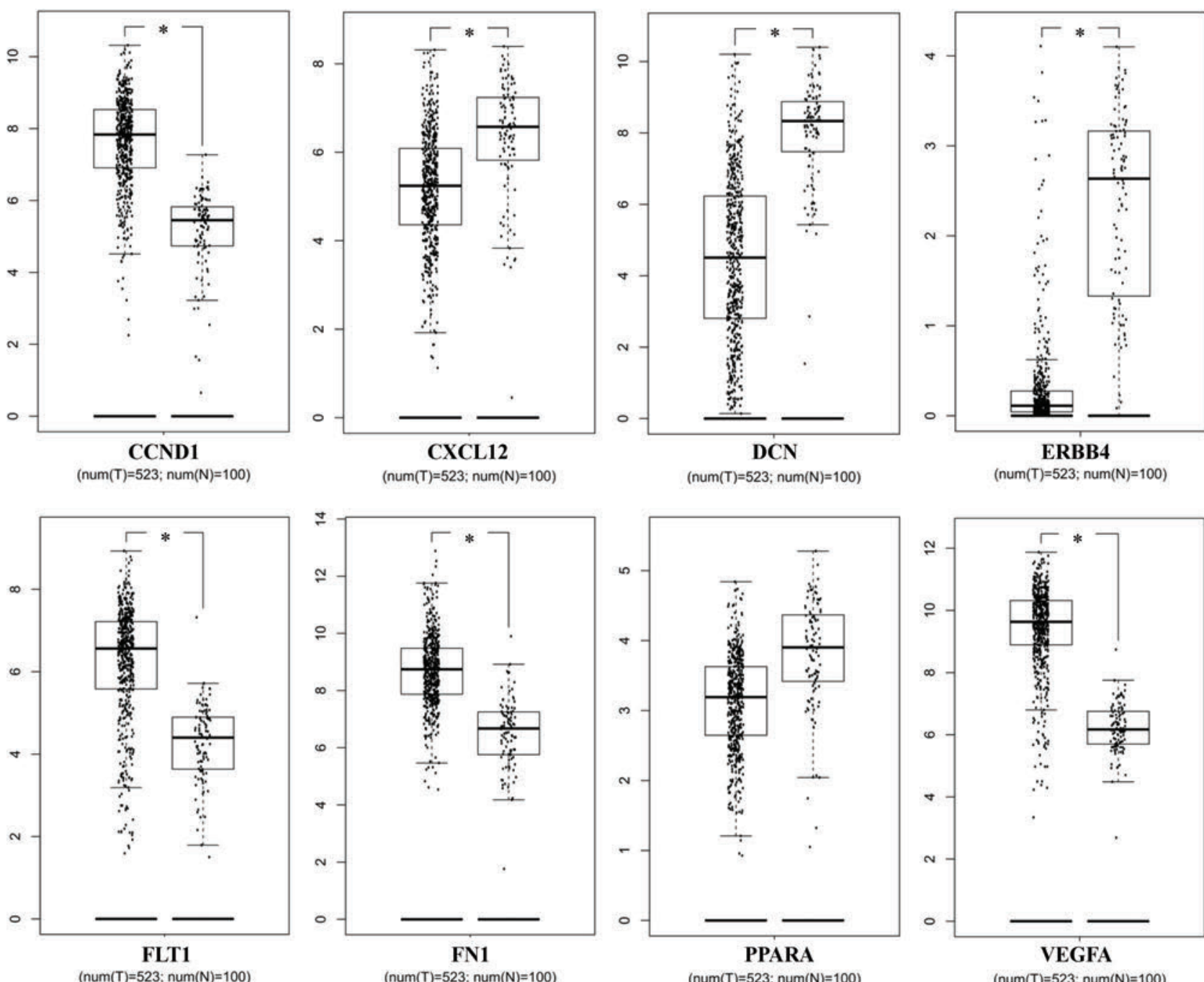

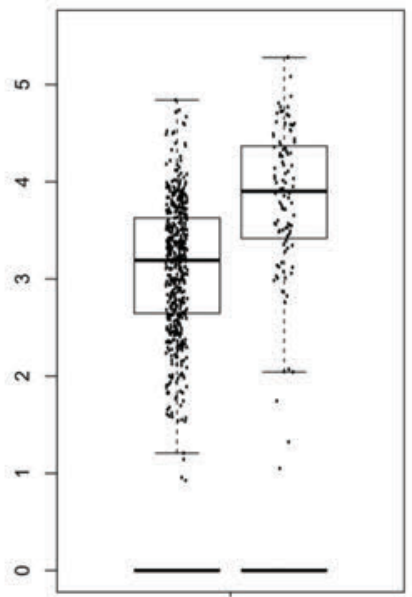

PPARA

(num $(T)=523 ; \operatorname{num}(\mathrm{N})=100$ )

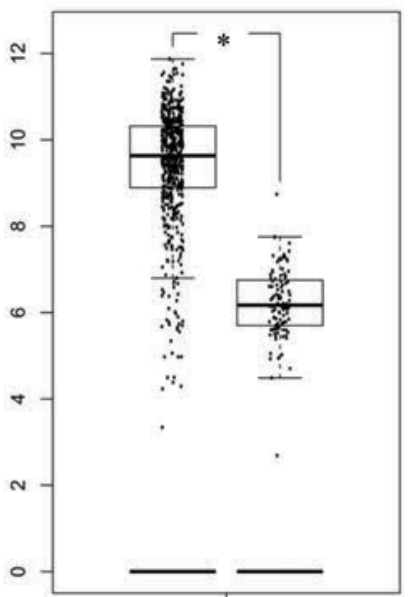

VEGFA

$(\operatorname{num}(\mathrm{T})=523 ; \operatorname{num}(\mathrm{N})=100)$

Figure 8. Boxplot of expression validation. ${ }^{*}$ : $\mathrm{p}<0.05$; T: tumor; N: normal

eration and migration of vascular endothelial cells, and is essential for both physiological and pathological angiogenesis. Burgesser et al. [22] reported that there is a direct linear relationship between expressions of VEGF-A and HIF- $1 \alpha$ in ccRCC samples. Besides, VEGFA can promote proliferation, migration and invasion, and suppress apoptosis of 786-O cells [23]. FLT1 is a member of the vascular endothelial growth factor receptor (VEGFR) family. Similarly, Flt1 promotes the growth and metastasis of tumor cells [24]. In addition, FLT1 can induce tumor metastasis [25]. Moreover, hypermethylated FLT1 plays an important role in chemotherapy resistance [26]. CXCL12 plays a role in many cellular functions, such as tumor growth and metastasis. CXCL12 was up-regulated in bladder cancer and related to the differentiation degree and invasive depth of cancer tissues [27]. DCN is an important component of the extracellular matrix. DCN underexpression is correlated with the presence of KRAS mutations [28].
Experimental validation was performed to confirm our results predicted by bioinformatics analysis. The $\mathrm{qPCR}$ results revealed that the expression of CCND1 and FN1 was higher in 786-O cell line than in HK-2 cell line, while the expression of ERBB4 and PPARA was lower in 786-O cell line than in HK-2 cell line. CCND1 is frequently overexpressed in early stages of gastric carcinoma [29]. It is the target of multiple biomarkers in different cancers, such as prostate cancer, bladder cancer and cervical cancer [30, 31]. FN1 is overexpressed in prostate cancer and related to aggressive prostate cancer [32]. However, there is no report about FN1 in ccRCC. As for ERBB4, it can directly regulate HIF-1 $\alpha$ and promote HIF-1a stability in a series of human cancers [33]. It has been found that PPARA mRNA level was decreased in hepatocellular carcinoma regulated by miR-9 [34]. Besides, PPARA physically bounds to Bcl 2 protein leading to reduced cancer cell chemoresistance [35]. 

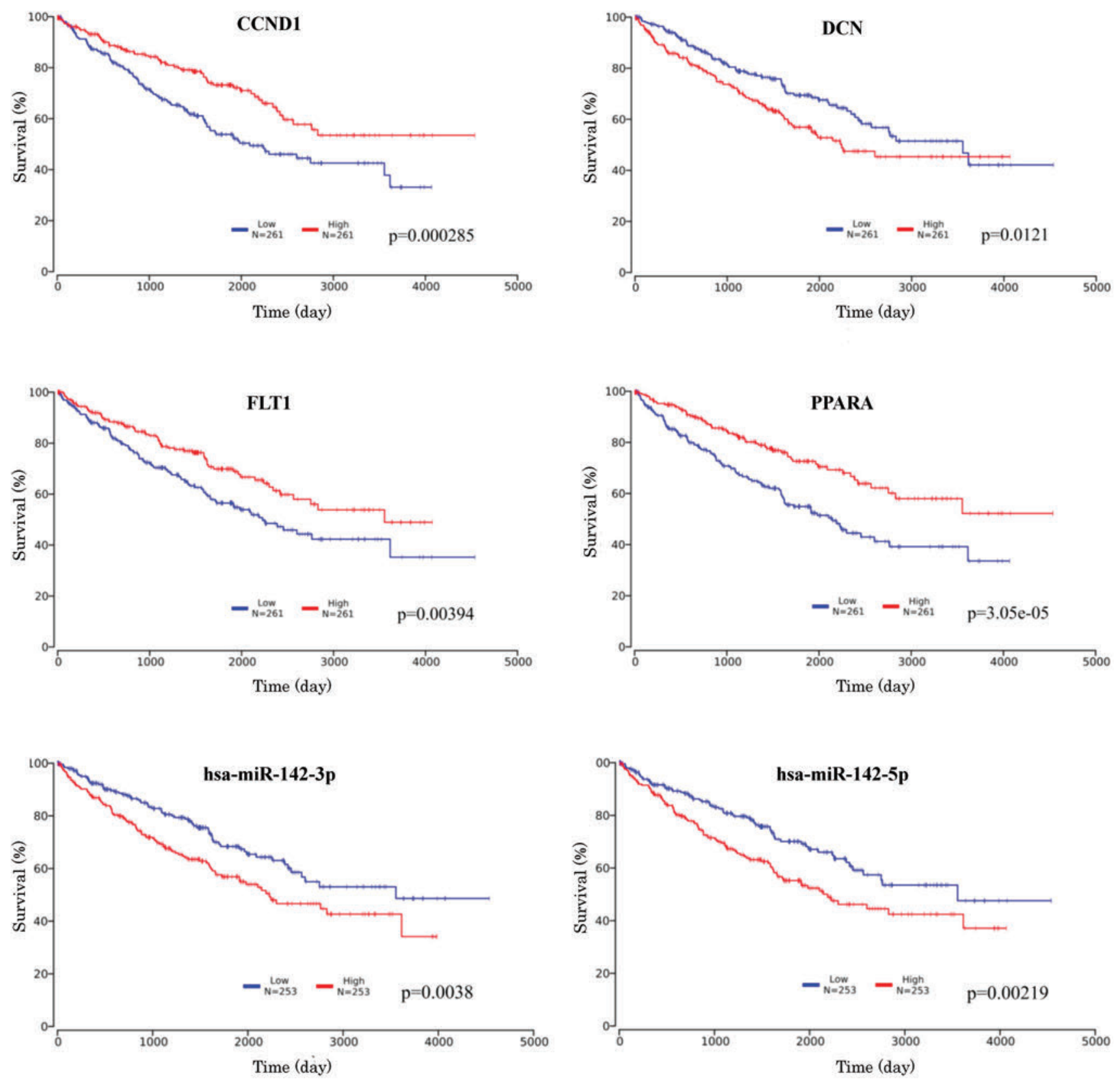

Figure 9. Survival analysis for mRNAs and miRNAs.

Multiple factors participate in the occurrence and progression of malignant tumors. By combined analysis of mRNAs, miRNAs, interactions and regulatory networks, the molecular mechanisms of tumors can be investigated and explained. This is a useful approach to identify biomarkers playing the most important role in cancer. In this study, we identified the biomarkers of ccRCC by bioinformatic analysis and performed experimental validation. However, there are still some limitations in this study. Due to the difficulty to obtain clinical samples and information, there was a lack of validation in tissue. Besides, we only checked the mRNA levels in one ccRCC cell line and one normal cell line, which cannot fully explain the different expression of biomarkers in ccRCC.

\section{Conclusion}

In our study, eight gene biomarkers were identified in ccRCC, including VEGFA, PPARA, CCND1, FLT1, CXCL12, FN1, DCN and ERBB4. Moreover, four biomarkers were 

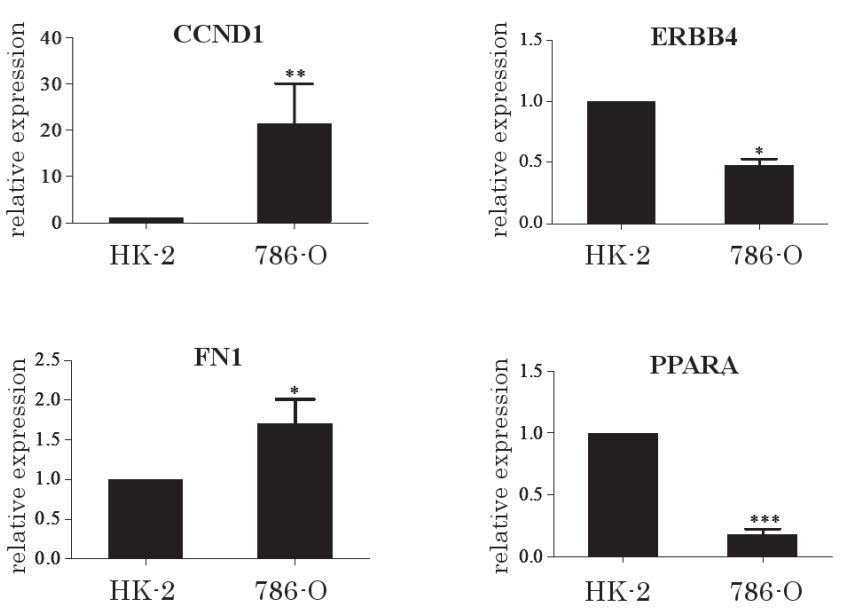

Figure 10. QPCR results for identified gene biomarkers. Expression of these DEGs was normalized against ACTB expression. The statistical significance of differences was calculated by Student's t test. ${ }^{*}: \mathrm{p}$-value $<0.05$, ${ }^{* *}$ : p-value $<0.01,{ }^{* *}$ : p-value $<0.001$

verified by qPCR. These genes might play important roles in the ccRCC research. Besides, a series of significant molecular mechanisms and pathways were provided for diagnosis and therapy.

\section{References}

[1] GRIGNON DJ, CHE M. Clear cell renal cell carcinoma. Clin Lab Med 2005; 25: 305-316. doi: 10.1016/j.cll.2005.01.012

[2] GIGLIONE P, LOMBARDO F, TOSCANI I, PAGLINO C, PORTA C. [Current approaches to the first-line treatment of clear-cell renal cell carcinoma]. Recenti Prog Med 2015; 106: 370-379. doi: 10.1701/1960.21302

[3] MAJER W, KLUZEK K, BLUYSSEN H, WESOLY J. Potential Approaches and Recent Advances in Biomarker Discovery in Clear-Cell Renal Cell Carcinoma. J Cancer 2015; 6: 1105-1113. doi: 10.7150/jca.12145

[4] SHIBASAKI N, YAMASAKI T, KANNO T, ARAKAKI R, SAKAMOTO $\mathrm{H}$ et al. Role of IL13RA2 in Sunitinib Resistance in Clear Cell Renal Cell Carcinoma. PLoS One 2015; 10:e0130980. doi: 10.1371/journal.pone.0130980

[5] SALAMA MF, CARROLL B, ADADA M, PULKOSKIGROSS M, HANNUN YA et al. A novel role of sphingosine kinase-1 in the invasion and angiogenesis of VHL mutant clear cell renal cell carcinoma. FASEB J 2015; 29: 2803-2813. doi: 10.1096/fj.15-270413

[6] XIANG W, HE J, HUANG C, CHEN L, TAO D et al. miR106b-5p targets tumor suppressor gene SETD2 to inactive its function in clear cell renal cell carcinoma. Oncotarget 2015; 6: 4066-4079. doi: 10.18632/oncotarget.2926

[7] KHELLA HW, SCORILAS A, MOZES R, MIRHAM L, LIANIDOU $E$ et al. Low expression of miR-126 is a prognostic marker for metastatic clear cell renal cell carcinoma. Am J Pathol 2015; 185: 693-703. doi: 10.1016/j.ajpath.2014.11.017
[8] THOMAS PD. The Gene Ontology and the Meaning of Biological Function. Methods Mol Biol 2017; 1446: 15-24. doi: 10.1007/978-1-4939-3743-1_2

[9] XING Z, CHU C, CHEN L, KONG X. The use of Gene Ontology terms and KEGG pathways for analysis and prediction of oncogenes. Biochim Biophys Acta 2016; 1860: 2725-2734. doi: 10.1016/j.bbagen.2016.01.012

[10] KHUNLERTGIT N, YOON BJ. Incorporating topological information for predicting robust cancer subnetwork markers in human protein-protein interaction network. BMC Bioinformatics 2016; 17: 351. doi: 10.1186/s12859016-1224-1

[11] CLOUGH E, BARRETT T. The Gene Expression Omnibus Database. Methods Mol Biol 2016; 1418: 93-110. doi: 10.1007/978-1-4939-3578-9_5

[12] LIVAK KJ, SCHMITTGEN TD. Analysis of relative gene expression data using real-time quantitative PCR and the 2(-Delta Delta C(T)) Method. Methods 2001; 25: 402-408. doi: 10.1006/meth.2001.1262

[13] XIE C, MAO X, HUANG J, DING Y, WU J et al. KOBAS 2.0: a web server for annotation and identification of enriched pathways and diseases. Nucleic Acids Res 2011; 39: W316322. doi: 10.1093/nar/gkr483

[14] BELOZEROV VE, VAN MEIR EG. Hypoxia inducible factor-1: a novel target for cancer therapy. Anticancer Drugs 2005; 16: 901-909.

[15] LISANTI MP, MARTINEZ-OUTSCHOORN UE, CHIAVARINA B, PAVLIDES S, WHITAKER-MENEZES D et al. Understanding the "lethal" drivers of tumor-stroma co-evolution: emerging role(s) for hypoxia, oxidative stress and autophagy/mitophagy in the tumor micro-environment. Cancer Biol Ther 2010; 10: 537-542. doi: 10.4161/cbt.10.6.13370

[16] BOROUGHS LK, DEBERARDINIS RJ. Metabolic pathways promoting cancer cell survival and growth. Nat Cell Biol 2015; 17: 351-359. doi: 10.1038/ncb3124

[17] HANAHAN D, WEINBERG RA. Hallmarks of cancer: the next generation. Cell 2011; 144: 646-674. doi: 10.1016/j. cell.2011.02.013

[18] KAMPHORST JJ, CROSS JR, FAN J, DE STANCHINA E, MATHEW R et al. Hypoxic and Ras-transformed cells support growth by scavenging unsaturated fatty acids from lysophospholipids. Proc Natl Acad Sci U S A 2013; 110: 88828887. doi: $10.1073 /$ pnas. 1307237110

[19] SHETA EA, TROUT H, GILDEA JJ, HARDING MA, THEODORESCU D. Cell density mediated pericellular hypoxia leads to induction of HIF-1alpha via nitric oxide and Ras/ MAP kinase mediated signaling pathways. Oncogene 2001; 20: 7624-7634. doi: 10.1038/sj.onc. 1204972

[20] BURGESSER MV, RIVA V, OJEDA SM, MUNOZ MORALES D, CALAFAT P et al. [Expression of VEGF-A, HIF$1 \mathrm{~A}, \mathrm{CD} 34$ and Ki67 in clear cell renal cell carcinomas and their relationship with conventional prognostic markers]. Rev Fac Cien Med Univ Nac Cordoba 2014; 71: 7-15.

[21] ZENG FC, ZENG MQ, HUANG L, LI YL, GAO BM et al. Downregulation of VEGFA inhibits proliferation, promotes apoptosis, and suppresses migration and invasion of renal clear cell carcinoma. Onco Targets Ther 2016; 9: 2131-2141. doi: 10.2147/OTT.S98002 
[22] ROYBAL JD, ZANG Y, AHN YH, YANG Y, GIBBONS DL et al. miR-200 Inhibits lung adenocarcinoma cell invasion and metastasis by targeting Flt1/VEGFR1. Mol Cancer Res 2011; 9: 25-35. doi: 10.1158/1541-7786.MCR-10-0497

[23] QIAN BZ, ZHANG H, LI J, HE T, YEO EJ et al. FLT1 signaling in metastasis-associated macrophages activates an inflammatory signature that promotes breast cancer metastasis. J Exp Med 2015; 212: 1433-1448. doi: 10.1084/jem.20141555

[24] KIM JY, HWANG J, LEE SH, LEE HJ, JELINEK J et al. Decreased efficacy of drugs targeting the vascular endothelial growth factor pathway by the epigenetic silencing of FLT1 in renal cancer cells. Clin Epigenetics 2015; 7: 99. doi: 10.1186/ s13148-015-0134-9

[25] YANG DL, XIN MM, WANG JS, XU HY, HUO Q et al. Chemokine receptor CXCR4 and its ligand CXCL12 expressions and clinical significance in bladder cancer. Genet Mol Res 2015; 14: 17699-17707. doi: 10.4238/2015.December.21.43

[26] KUMARI S, PUNEET, PRASAD SB, YADAV SS, KUMAR $\mathrm{M}$ et al. Cyclin D1 and cyclin E2 are differentially expressed in gastric cancer. Med Oncol 2016; 33: 40. doi: 10.1007/ s12032-016-0754-8

[27] YIN H, SHENG Z, ZHANG X, DU Y, QIN C et al. Overexpression of SOX18 promotes prostate cancer progression via the regulation of TCF1, c-Myc, cyclin D1 and MMP-7. Oncol Rep 2017; 37: 1045-1051. doi: 10.3892/or.2016.5288

[28] SHIN SS, PARK SS, HWANG B, MOON B, KIM WT et al. MicroRNA-892b influences proliferation, migration and invasion of bladder cancer cells by mediating the p19ARF/cyclin D1/CDK6 and Sp-1/MMP-9 pathways. Oncol Rep 2016; 36: 2313-2320. doi: 10.3892/or.2016.5052

[29] DAS DK, OGUNWOBI OO. A novel microRNA-1207-3p/ FNDC1/FN1/AR regulatory pathway in prostate cancer. RNA Dis 2017; 4. pii: e1503
[30] PAATERO I, JOKILAMMI A, HEIKKINEN PT, ILJIN K, KALLIONIEMI OP et al. Interaction with ErbB4 promotes hypoxia-inducible factor-1alpha signaling. J Biol Chem 2012; 287: 9659-9671. doi: 10.1074/jbc.M111.299537

[31] DRAKAKI A, HATZIAPOSTOLOU M, POLYTARCHOU C, VORVIS C, POULTSIDES GA et al. Functional microRNA high throughput screening reveals miR-9 as a central regulator of liver oncogenesis by affecting the PPARA-CDH1 pathway. BMC Cancer 2015; 15: 542. doi: 10.1186/s12885015-1562-9

[32] GAO J, LIU Q, XU Y, GONG X, ZHANG R et al. PPARalpha induces cell apoptosis by destructing Bcl2. Oncotarget 2015; 6: 44635-44642. doi: 10.18632/oncotarget.5988

[33] MARTINEZ-OUTSCHOORN UE, BALLIET RM, RIVADENEIRA DB, CHIAVARINA B, PAVLIDES S et al. Oxidative stress in cancer associated fibroblasts drives tumorstroma co-evolution: A new paradigm for understanding tumor metabolism, the field effect and genomic instability in cancer cells. Cell Cycle 2010; 9: 3256-3276. doi: 10.4161/ cc.9.16.12553

[34] TANG Z, LI C, KANG B, GAO G, LI C et al. GEPIA: a web server for cancer and normal gene expression profiling and interactive analyses. Nucleic Acids Res 2017. doi: 10.1093/ nar/gkx247

[35] MLAKAR V, BERGINC G, VOLAVSEK M, STOR Z, REMS $\mathrm{M}$ et al. Presence of activating KRAS mutations correlates significantly with expression of tumour suppressor genes DCN and TPM1 in colorectal cancer. BMC Cancer 2009; 9: 282. doi: 10.1186/1471-2407-9-282 